\title{
Acceptability of Provider Initiated HIV Counseling and Testing among Tuberculosis and Non-tuberculosis Patients in Shashemene Town, Ethiopia
}

\author{
Emiru Adeba $^{1^{\star}}$ and Wakgari Deressa ${ }^{2}$
}

\author{
${ }^{1}$ Department of Public Health, College of Medical and Health Sciences, Wollega University, P.O Box: 395, \\ Nekemte, Ethiopia \\ ${ }^{2}$ School of Public Health, Addis Ababa University, P.O Box: 11950, Addis Ababa, Ethiopia
}

\begin{tabular}{|c|c|}
\hline $\mathrm{Ab}$ & nation \\
\hline \multirow{10}{*}{$\begin{array}{l}\text { The objective of the study was to assess the acceptability of Provider Initiated HIV Counseling } \\
\text { and Testing (PIHCT) and factors influencing its service uptake among TB and non TB patients } \\
\text { in Shashemene town of West Arsi Zone. Institution-based, comparative cross-sectional study } \\
\text { was conducted from January to February } 2010 \text {, on } 237 \text { TB patients attending Tuberculosis } \\
\text { clinics and } 236 \text { non TB patients who were offered PIHCT from outpatient department of } \\
\text { Shashemene Hospital and Health Center. Data were collected using consecutive sampling } \\
\text { until the required sample size was attained using structured questionnaire. Majority of the } \\
\text { participants were male } 286(60.5 \%) \text {, in the age group } 25-34 \text { years } 172(36.4 \%) \text {, Muslim by } \\
\text { religion } 233(49 \%) \text {.Among } 473 \text { study participants } 86.3 \%(89.9 \% \text { TB versus } 82.6 \% \text { non TB) } \\
\text { had accepted PIHCT. TB patients are more likely to accept PICHT than non TB patients at } \\
\text { AOR= } 2.6 ; 95 \% \text { CI (1.3, } 5.0) \text {. Those who support importance of PIHCT were more likely to } \\
\text { accept PIHCT at AOR=11.4; } 95 \% \text { CI ( } 5.1,25.4) \text { than those who are against PIHCT. The } \\
\text { acceptance rate of PIHCT is relatively higher in this study; TB patients were more likely to } \\
\text { accept PIHCT than non TB patients. The programme needs to be strengthened in all settings } \\
\text { for both TB and non TB patients since HIV testing and counseling stands out as paramount } \\
\text { both in treatment and in prevention of HIV/AIDS. }\end{array}$} & \\
\hline & Revised \\
\hline & :epted : $26-03$ \\
\hline & \\
\hline & \\
\hline & PIHCT \\
\hline & TB and non TB \\
\hline & \\
\hline & \\
\hline & \\
\hline
\end{tabular}

\section{INTRODUCTION}

Tuberculosis (TB) is a leading cause of morbidity and mortality among people living with HIV/AIDS (PLWHA) (CDC, 2006). Untreated human immuno-deficiency virus (HIV) infection leads to progressive immunodeficiency, and increased susceptibility to infections including TB (Coetzee et al., 2004). HIV is fuelling the tuberculosis epidemic particularly in Africa, where TB incidence rates are still rising across the continent at a rate of $3-4 \%$ annually (WHO, 2000). TB had tripled or quadrupled in Sub-Saharan Africa (SSA) due to the deadly synergy effect between TB and HIV, since 1990 (WHO, 2005).

HIV infection is now the most important single predictor of TB incidence in SSA. The region accounts for $70 \%$ of the world's 14 million people who are co-infected with TB and HIV (WHO, 2004). Death rate in patients treated for tuberculosis in sub-Saharan Africa has risen in the last 10-15 years, the most important reasons being concomitant HIV infection (Ya Duil, Dermot and Anthony 2001). Between $20 \%$ and $30 \%$ of HIV infected patients with smear positive pulmonary tuberculosis $\left(\mathrm{PTB}^{+}\right)$die within 12 months of starting treatment. In Ethiopia, it is estimated that HIV/AIDS accounts for $32 \%$ of the estimated 141,000 TB case incidences in $2005(\mathrm{MOH}$, 2004). The prevalence of HIV co-infection among adult TB cases is estimated to be $40 \%$ in urban areas of Ethiopia (MOH, 2004). A study conducted in Addis Ababa in 1998 showed HIV co infection was 45.3\% among PTB+ patients (Demissie, Lindtjorn and Tegbaru, 2004).

Despite close epidemiological links between HIV and $\mathrm{TB}$, the public health responses have largely been separated (WHO, 2004). In addition, because of the low priority given to voluntary counseling and testing (VCT) in the past, access to this service has been limited in countries most severely affected by HIV (Fylkesnes and siziya, 2004). According to the $2003 \mathrm{WHO}$ report, only $3 \%$ of the 4.4 million TB cases were reported to have been HIV tested worldwide (WHO, 2009).

Due to the under utilization of the client initiated HIV counseling and testing the revised UNAIDS / WHO policy statement on HIV testing recommended that PIHCT should be implemented in clinical settings (WHO, 2005). For instance, only $10 \%$ of patients knew their sero-status in the world in 2003 (Knut, Alan, Catharina and Pascal, 1999). In Ethiopia, the second round behavioral surveillance survey (BSS) reported that only $5 \%$ of the general population (15-49 years of age) being ever tested for HIV in 2005 (MOH, 2006).

HIV pandemic presents a massive challenge to the control of TB (UNAIDS, 2004). On the other hand, TB is also one of the leading causes of morbidity and mortality 


\section{Emiru Adeba and Wakgari Deressa}

in PLWHA in Ethiopia (MOH, 2003). HIV testing and counseling stands out as paramount to tackle these problems in treatment and prevention activities. Results of VCT acceptability assessments in the general population as well as among TB patients showed wide variability. The National guideline for management of opportunistic infections and ART in adolescents and adults in Ethiopia recommends HIV counseling and testing as a routine care for TB patients (MOH, 2007). Shashemene City Administration Health Bureau is implementing PIHCT in response to the high HIV prevalence among TB patients, and in an attempt to increase the uptake of HIV testing and antiretroviral treatment (ART).

\section{MATERIAL AND METHODS}

\section{Study Area and Population}

The study was conducted in Shashemene town, which is located 250 kilometers south of the capital city, Addis Ababa, situated at a crossroad to Bale, Arsi, Zeway, Awasa and most parts of Southern Ethiopia. The study was conducted from January 01 to Feburary 30, 2010. According to the Central Statistics Authority 102,000 people (51\% female and $49 \%$ male) were estimated to live in Shashemene town in 2007 (37). The town is divided in to 10 kebeles (the smallest administrative units) with an average population of over 10,000 in each kebele (ACORD, 1999-2003).

\section{Study Design}

A facility-based, comparative cross-sectional study was conducted among TB patients attending their treatment under DOTS strategy and non TB patients from the health institution who were offered with PIHCT. For this study, the source population was all TB patients who were registered under DOTS and non TB patients who were offered PIHCT during the study period in Shashamane hospital and health center, West Arsi Zone.

\section{Sample Size Determination and Sampling Procedure}

The sample size for this study was determined using the formula for the difference between population proportions. The number of population willing to accept PIHCT was the main outcome variable used to calculate the sample size and a study conducted in Arbaminch Town shows the prevalence of willingness to accept PIHCT was $35 \%$ among cases (Jerene, Endale and Lindtjorn, 2007) and 50\% among controls were assumed to consider maximum sample size since there is no similar study done, with 5\% marginal error and $95 \%$ confidence interval, and $80 \%$ power. Assuming $15 \%$ non-response rate, the minimum sample size was calculated to be 473 for both groups using the formula for difference between population proportions.

Data were collected from the all government health facilities, both hospital and health centers where DOTS are being implemented in Shashemene town i.e. Hospital and health center in Shashemene town over the data collection period. Patients who visited the health facilities and fulfilled the inclusion criteria during the study period for treatment of TB and patients who initiated PIHCT at the outpatient departments were interviewed consecutively until the total sample size of the study was completed. The sample size was proportionally allocated to Shashemene hospital and health center based on monthly client size. Study subjects in the selected institutions were stratified by patient status related to presence or absence of TB and sample size for each stratum was equally allocated. To select study subjects
Sci. Technol. Arts Res. J., Jan-March 2014, 3(1): 116-121

within each stratum quota sampling was used. Initially the daily average number of clients visiting the health institution during data collection period was estimated based on the previous daily client flow of the units. This was obtained by referring client registration book/ record for a month prior to data collection. The study utilized closed ended structured questionnaire containing questions on socio demographic variables, patients' knowledge and attitude related to TB/HIV/AIDS, self risk perception to HIV/AIDS and knowledge and attitude related to PIHCT. The questionnaire was prepared in English and then translated in to the local language (Afan Oromo) and then back translated into English to check for its consistency.

\section{Data Processing and Analysis}

EPI INFO version 6 was used for data entry and cleaning, and SPSS16 for Statistical analysis. In the analysis process, frequency distributions of variables were worked out in order to describe them in relation with the study population. Knowledge on major HIV/AIDS transmission and prevention was weighted for 4 and 3 questions; score greater than $75 \%$ ( 3 and above out of 4 ) and $67 \%$ (2 and above out of 3 ) are defined for existence of adequate knowledge. And again, for HIV/AIDS comprehensive knowledge five variables were measured, namely, existence of disease, weighted mode of transmission, weighted means of prevention, nature of the disease and curability. An average score greater than $80 \%$ are defined as presence of adequate comprehensive HIV/AIDS knowledge

\section{Ethical Clearance}

Ethical clearance was obtained from the Institutional Review Board of the Faculty of Medicine at Addis Ababa University. Permission to undertake the study was obtained from Oromia Regional State Health Bureau and from administrative bodies of Shashemene town including West Arsi Zone Health Bureau. Informed written consent was obtained from all consented study participants.

\section{RESULTS}

\section{Socio Demographic Characteristics of Participants}

A total of $237(50.2 \%)$ TB and $236(49.8 \%)$ non TB patients participated in the study. About $68 \%$ (323) of the participants were from Shashemene hospital, while the remaining 150(32\%) were from Shashemene health center. Fifty seven percent male and $42.6 \%$ female TB and $63.6 \%$ male and $36.4 \%$ female non TB patients were participated in the interview respectively. The mean and median ages of the patients were 30 and 27 years old for TB patients and 30 and 28 years old for non TB patients, respectively.

Nearly half $(48.9 \%)$ of the respondents were Muslims; followed by, Orthodox Christians $76(32.1 \%$ ) among TB patients and similarly $49.2 \%$ Muslim followed by Orthodox Christian $53(22.5 \%)$ among non TB patients by religion. About $57 \%$ and $64.8 \%$ of the study participants were from Oromo ethnic group followed by Amhara, 41(17.3\%) and $37(15.7 \%)$ among TB and non TB patients respectively. The rest were Gurage, and few others.

Regarding the marital status of the participants, $113(47.7 \%)$ were single, $102(43 \%)$ was married in union, and $5.5 \%$ were widowed among TB patients. While $149(63.1 \%)$ were married in union, $83(35.2 \%)$ were single and $2(0.8 \%)$ were widowed from non TB patients. The educational status of the study group is similar both in TB 


\section{Emiru Adeba and Wakgari Deressa}

and non TB patients with 97(40.9\%) and 127(53.8\%) completed greater than grade eight, $75(31.6 \%)$ and $63(26.7 \%)$ completed grade five to eight, $33(13.9 \%)$ and $30(12.7 \%)$ read and write up to grade four and $33(13.9 \%)$ and $30(12.7 \%)$ are illiterate respectively. Majority of the participants were students $63(26.6 \%)$ and $56(23.7 \%)$ followed by civil servants $32(13.5 \%)$ and $42(17.8 \%)$ both in TB and non TB patients respectively. Others including merchants, daily laborer, house wife and domestic servants are also participated in both groups. Of the total participants responded to the income variable 65(27.5\%) and $50(21 \%)$ were reported that they have monthly income of less than 400 Ethiopian birr and 49(20.7) and $73(30.8 \%)$ have a monthly income of greater than 400 Ethiopian birr among TB and non TB patients respectively. Thirty four percent of TB and $59(25 \%)$ of non TB patients were not responded to income variables.

\section{Knowledge and Attitude of Participants toward PIHCT}

Nearly all $(96 \%)$ of the study participants were aware of the availability of PIHCT in health institutions (94.1\% TB Vs $97.9 \%$ non TB). The most common sources of information about PIHCT for the participants were health worker/institutions as mentioned by $89 \%$ of TB and $91.1 \%$ of non TB patients, mass media by $74.3 \%$ of TB patients
Sci. Technol. Arts Res. J., Jan-March 2014, 3(1): 116-121

and $84.7 \%$ of non TB patients followed by friends by $22.4 \%$ of TB and $54.2 \%$ non TB patients and family members by $20.3 \%$ TB $45.3 \%$ of non TB patients. Majority of the patients have positive attitude toward PIHCT after it was explained to them by their treatment supervisor. $86.6 \%$ TB and $70.8 \%$ non TB patients were "extremely" in favor of PIHCT. $96.2 \%$ of TB and $98.3 \%$ of non TB patients agreed that any one should check his/her HIV sero status.

Most of the participants both from TB and non TB patients $(95.8 \%$ and $98.7 \%)$ respectively believed that PIHCT is important. Among those who believe PIHCT is important, majority of them agreed that PIHCT result in gain access to ART $(72.2 \%, 76.3 \%)$, helps to get care and support if positive $(73.8 \%, 91.5 \%)$, makes it easier for them to get tested $(77.6 \%, 78.8 \%)$, and helps to protect infection $(81.9 \%, 91.9 \%)$ by TB and non TB patients respectively. On the other hand, among 56(23.6\%) TB and $13(5.5 \%)$ non TB patients, who believe that PIHCT has influence on TB treatment service utilization, $39(16.5 \%)$ TB and $11(4.7 \%)$ non TB believed that PIHCT would cause TB patients to avoid seeing health care provider for fear of being tested (table 1).

Table 1: Knowledge and Attitude Related to PIHCT among TB and non-TB Patients.

\begin{tabular}{lccc}
\hline \multirow{2}{*}{ Variables } & TB patients & Non TB patients & Both TB and non TB patients \\
\cline { 2 - 4 } & Number (\%) & Number (\%) & Number (\%) \\
\hline Source of information* & & & \\
Health worker/institution & $211(89)$ & $215(91.1)$ & $426(90)$ \\
Mass media & $176(74.3)$ & $200(84.7)$ & $376(79.5)$ \\
Friends & $53(22.4)$ & $128(54.2)$ & $181(38.3)$ \\
Family members & $48(20.3)$ & $107(45.3)$ & $155(32.7)$ \\
\hline In favor of PIHCT & & \\
Extremely & $205(86.5)$ & $167(70.8)$ & $372(78.6)$ \\
Very much & $12(5.9)$ & $58(25.8)$ & $70(14.8)$ \\
To some extent and not at all & $20(7.6)$ & $11(3.4)$ & $31(6.6)$ \\
\hline Reasons for PIHCT is important * & & & \\
To know their HIV sero status & $184(77.6)$ & $186(78.8)$ & $370(78)$ \\
To protect infection & $194(81.9)$ & $217(91.9)$ & $311(86.8)$ \\
If positive, not to transmit to others & $184(77.6)$ & $195(82.6)$ & $391(80)$ \\
If positive ,to get support and care & $175(73.8)$ & $216(91.5)$ & $351(74.2)$ \\
If positive ,to get ART & $171(72.2)$ & $180(76.3)$ & $273(57.7)$ \\
To be free from stress & $124(52.3)$ & $149(63.1)$ & \\
& ${ }^{*}$-more than one response is possible
\end{tabular}

\section{Acceptability of PIHCT and Factors Associated with its Utilization}

This study assessed factors associated with acceptability of PIHCT. A logistic regression model was used to examine factors associated with acceptability of PIHCT (having been tested for HIV following supervisor initiation) as dependent variable. A stepwise forward regression analysis was utilized in three steps in order to find out predictors on acceptability of PIHCT. The first step was related to the socio demographic variables, in the second step knowledge; attitude and risk perception towards TB/ HIV/AIDS was involved, and the last step involved analyzing the joint effects of the variables on socio-demographic, knowledge and self risk perception on acceptability of PIHCT in order to detect weak associations that may be strong during interactions with other Variables. Variables with $P<0.05$ were considered for further analysis.

\section{Socio Demographic Determinants of Acceptability of PIHCT}

The association between socio demographic characteristics of TB and non TB patients with acceptability of PIHCT was assessed using Univariate and multivariate logistic regression models. In the bivariate analysis, being younger, education status of grade 5-8, being male and TB patient were more likely associated with acceptance of PIHCT. Controlling for other variables in the logistic regression model, being TB patient was strongly associated with acceptance of PIHCT (table 2).

\section{Association of Socio-demographic Characteristics, Knowledge and Attitude with Acceptance of PIHCT}

Knowledge related factors like believing that HIV is not curable illness $(p=0.6)$, having ever heard of the disease called HIV/AIDS ( $p=0.7)$, believing that one self can be infected with HIV/AIDS $(p=0.2)$, were not significantly associated with acceptability of PIHCT for the overall study participants.

Those believing that HIV infection could be asymptomatic $[\mathrm{COR}(95 \% \mathrm{Cl}=2.4(1.2,4.8)]$, patients those who believed that PIHCT is important [COR $(95 \% \mathrm{Cl}$ $=13.5(6.2,28.9)]$, believing that control of TB helps to control HIV/AIDS [COR $(95 \% \mathrm{Cl}=1.9(1.1,3.4)]$ and knowing 


\section{Emiru Adeba and Wakgari Deressa}

any one infected or died of HIV/AIDS [COR $(95 \% \mathrm{Cl}=3.8$ $(2.2,6.6)]$ were significantly associated with PIHCT acceptance in bivirate analysis for the overall study subjects. After variables were controlled for confounding factors using multiple logistic regression analysis only those patients who feel PIHT is important and those who have known someone with/died of HIV/AIDS remain associated with acceptance of PIHCT in the overall study participants

Among all, those respondents who agree PIHCT is important are nearly eleven times more likely to accept PIHCT when compared to those who were against PIHCT [Adjusted $\mathrm{OR}=10.7 ; 95 \% \mathrm{Cl}(4.2,27.3)$, those who have known someone with or died of HIV/AIDS are three times more likely to accept PIHCT than those who haven't [Adjusted $\mathrm{OR}=3.4 ; 95 \% \mathrm{Cl}(1.0,11.7)$ and TB patients are twice as more likely to accept PIHCT than non TB patients [Adjusted $\mathrm{OR}=2.6 ; 95 \% \mathrm{Cl}(1.3,5.0)$. The other variables were not significantly associated with PIHCT acceptability after adjusting for confounders (table 3 ).

\section{DISCUSSION}

More than half $(57.8 \%$ TB and $56.4 \%$ non TB) of the study participant were young age of $<29$ years old. This might be due the high prevalence of HIV/AIDS infection in this age group which could have made them susceptible to TB. The findings of this study (high prevalence of TB occurred in the young $(<29)$ population) is similar to
Sci. Technol. Arts Res. J., Jan-March 2014, 3(1): 116-121

findings documented by several studies and the WHO (Jerene, Endale and Lindtjorn, 2007).

Assessment of high-risk behaviors in Ethiopia was initiated in the 1990s. High scores especially in knowledge of mode of viral transmission were documented which showed some success in raising awareness in the general population and sub-groups. Adequate knowledge on viral transmission and prevention is believed to be a key factor in fighting the epidemic. Regarding the findings of the respondents' knowledge on mode of HIV transmission and prevention, most of the participants had the correct knowledge. In this study a substantial proportion of TB patients (88.4\%) and non TB patients (87.3\%) were aware of asymptotic healthy carriers can be HIV case, knowledge on mother-to-child transmission [during pregnancy $(56.5 \%)$ for TB and $(74.2 \%)$ for non TB patients and via breast feeding (84.4\%) for TB and $(71.2 \%)$ for non TB patients] was found relatively higher compared to study done in other area On the other hand misconceptions regarding HIV transmission like transmission through sharing meal with HIV infected person and mosquito bite $15.8 \%$ in TB and $10 \%$ in non TB patients) was relatively higher than similar study done in Adama (3.7\%). Misconceptions on HIV transmission and poor knowledge about the disease would cause stigma associated with the disease that have impacts on the control of the epidemic (Seid, 2008; Higgins, 1991).

Table 2: Socio-demographic variables of TB and non-TB Patients and acceptability of PIHCT.

\begin{tabular}{|c|c|c|c|c|}
\hline \multirow{2}{*}{ Variables } & \multicolumn{2}{|c|}{ PIHCT } & \multicolumn{2}{|l|}{$95 \% \mathrm{Cl}$} \\
\hline & Acceptor & Non acceptor & COR(95\% Cl) & AOR(95\% Cl) \\
\hline \multicolumn{5}{|l|}{ Age(years) } \\
\hline $15-24$ & 152 & 20 & $2.2(1.0,4.9)$ & $1.6(0.5,4.8)$ \\
\hline $25-34$ & 151 & 21 & $2.1(0.9,4.6)$ & $1.9(0.7,4.7)$ \\
\hline $35-44$ & 64 & 12 & $1.5(0.6,3.8)$ & $1.7(0.6,4.8)$ \\
\hline$>45$ & 41 & 12 & 1.0 & 1.0 \\
\hline \multicolumn{5}{|l|}{ Sex } \\
\hline Male & 250 & 36 & $1.2(0.7,2.1)$ & $1.3(0.6,2.6)$ \\
\hline Female & 158 & 29 & 1.0 & 1.0 \\
\hline \multicolumn{5}{|l|}{ Religion } \\
\hline Orthodox & 113 & 16 & 1.0 & 1.0 \\
\hline Muslim & 202 & 30 & $0.9(0.4,1.8)$ & $0.9(0.4,2.4)$ \\
\hline Protestant & 61 & 14 & $0.6(0.2,1.3)$ & $0.8(0.3,2.3)$ \\
\hline Catholic & 23 & 1 & $3.2(0.4,25.7)$ & $0.4(0.1,1.8)$ \\
\hline \multicolumn{5}{|l|}{ Ethnicity } \\
\hline Oromo & 253 & 35 & 1.0 & 1.0 \\
\hline Amhara & 69 & 9 & $1.0(0.4,2.3)$ & $0.8(0.3,2.4)$ \\
\hline Gurage & 42 & 4 & $1.4(0.4,4.2)$ & $1.3(0.4,4.5)$ \\
\hline Tigre & 19 & 4 & $0.6(0.2,2.0)$ & $0.3(0.1,1.5)$ \\
\hline \multicolumn{5}{|l|}{ Marital status } \\
\hline Married & 213 & 38 & 1.0 & 1.0 \\
\hline Single & 195 & 27 & $1.2(0.7,2.1)$ & $0.6(0.3,1.3)$ \\
\hline \multicolumn{5}{|l|}{ Patient status } \\
\hline TB patient & 213 & 24 & $1.8(1.0,3.2)$ & $1.9(1.0,3.6)$ \\
\hline No TB patient & 195 & 41 & 1.00 & 1.0 \\
\hline \multicolumn{5}{|l|}{ Educational level } \\
\hline Illiterate & 36 & 12 & 1.0 & 1.0 \\
\hline completed grade 4 & 51 & 12 & $1.4(0.5,3.5)$ & $0.9(0.3,2.5)$ \\
\hline Completed grade 5-8 & 127 & 11 & $3.8(1.5,9.4)$ & $2.3(0.8,6.5)$ \\
\hline Greater than grade 8 & 194 & 30 & $2.1(1.0,4.6)$ & $0.9(0.3,2.7)$ \\
\hline \multicolumn{5}{|l|}{ Occupation } \\
\hline Civil servant & 68 & 6 & 1.0 & 1.0 \\
\hline Student & 107 & 12 & $0.7(0.2,2.1)$ & $0.6(0.1,2.2)$ \\
\hline Merchant & 69 & 11 & $0.5(0.1,1.5)$ & $0.3(0.1,1.1)$ \\
\hline House wife & 46 & 11 & $0.3(0.1,1.0)$ & $0.2(0.1,0.9)$ \\
\hline Daily laborer & 35 & 4 & $0.7(0.2,2.9)$ & $0.5(0.1,2.7)$ \\
\hline No job & 33 & 5 & $0.5(0.1,2.0)$ & $0.4(0.1,1.8)$ \\
\hline
\end{tabular}


Table 3: Proportion of acceptors and non acceptors of PIHCT and odds ratios $(95 \% \mathrm{Cl})$ from binary logistic regression assessing the association of PIHCT acceptance and selected variables among TB and non TB.

\begin{tabular}{|c|c|c|c|c|}
\hline \multirow{2}{*}{ Variables } & \multicolumn{2}{|c|}{$\begin{array}{l}\text { PIHCT } \\
\end{array}$} & \multicolumn{2}{|c|}{$95 \% \mathrm{Cl}$} \\
\hline & Acceptor & Non- acceptor & COR(95\% Cl) & AOR(95\% Cl) \\
\hline \multicolumn{5}{|l|}{ Age(years) } \\
\hline $15-24$ & 152 & 20 & $2.2(1.0,4.9)$ & $1.6(0.5,4.8)$ \\
\hline $25-34$ & 151 & 21 & $2.1(0.9,4.6)$ & $1.9(0.7,4.7)$ \\
\hline $35-44$ & 64 & 12 & $1.5(0.6,3.8)$ & $1.7(0.6,4.8)$ \\
\hline$>45$ & 41 & 12 & 1.0 & 1.0 \\
\hline \multicolumn{5}{|l|}{ Sex } \\
\hline Male & 250 & 36 & $1.2(0.7,2.1)$ & $1.3(0.6,2.6)$ \\
\hline Female & 158 & 29 & 1.0 & 1.0 \\
\hline \multicolumn{5}{|l|}{ Patient status } \\
\hline TB patient & 213 & 24 & $1.8(1.0,3.2)$ & $2.6(1.3,5.0)$ \\
\hline Non TB patient & 195 & 41 & 1.00 & 1.0 \\
\hline \multicolumn{5}{|c|}{ Health looking person be infected with HIV? } \\
\hline Yes & 367 & 51 & $2.4(1.2,4.8)$ & $1.8(0.8,3.8)$ \\
\hline No & 41 & 14 & 1.0 & 1.0 \\
\hline \multicolumn{5}{|l|}{ PIHCT is important } \\
\hline Yes & 395 & 45 & $13.5(6.2,28.9)$ & $11.4(5.1,25.4)$ \\
\hline No & 13 & 20 & 1.0 & 1.0 \\
\hline \multicolumn{5}{|c|}{ Control of TB helps control HIV/AIDS? } \\
\hline Yes & 315 & 41 & $1.9(1.1,3.4)$ & $1.2(0.6,1.8)$ \\
\hline No & 93 & 24 & 1.0 & 1.0 \\
\hline \multicolumn{5}{|c|}{ TB patients increased after HIV/AIDS? } \\
\hline Yes & 306 & 40 & $1.8(1.0,3.7)$ & $0.9(0.4,1.8)$ \\
\hline No & 102 & 25 & 1.0 & 1.0 \\
\hline \multicolumn{5}{|l|}{ know PLWHA } \\
\hline Yes & 304 & 24 & $3.8(2.2,6.6)$ & $3(1.6,5.5)$ \\
\hline No & 104 & 37 & 1.0 & 1.0 \\
\hline
\end{tabular}

The overall PIHCT acceptability rate of $86.3 \%$ of this study was slightly higher than a study conducted in Adama (81.2\%). In the present study the high prevalence of PICHT acceptability could be due to the availability of comprehensive HIV/AIDS care (availability of many free standing VCT centers, increased access to ART, OI and care and support services). Moreover, high knowledge of TB and HIV association could be the possible explanations for the relatively high prevalence of HIV test for the TB patients. It can be noted that a high theoretical acceptability which may not necessarily true for the high practical acceptability. A study conducted in Arbaminch (35\%) and Addis Ababa (67\%) also demonstrated a low prevalence of practical acceptability of PIHCT (Seid, 2007; Girma, 2008).

In pilot and clinical trials, when HIV counseling and testing was routinely offered by health care providers, the acceptability rate of HIV testing is reported to be satisfactorily high, reaching $90-100 \%$ for patients attending TB clinics in several countries. For instance, acceptability of HIV testing among TB patients was $91 \%$ in Guyana and Mali and 99\% in South Africa. Prospective study by Fisher et al showed that intention did predict performing specific HIV-preventive behavior under study. Therefore, the findings of the present study could be considered as indication of the need for promotion and expansion of PIHCT service to the public at large (Zachariah, 2003; Hisler, 2003).

There was widespread support for PIHCT, with $86.6 \%$ TB and $70.8 \%$ of non TB patients reporting that they were either extremely or very much in favor of PIHCT. Majority of respondents felt that PIHCT would increase uptake of ARV, number of tested patients and would decrease HIV related stigma. Patient who believed that PIHCT is important $\mathrm{OR}=11.4 ; 95 \% \mathrm{Cl}(5.1,25.4)$ and those who knows people living with HIV/AIDS OR=3; $95 \% \mathrm{Cl}$ (1.6,
5.5) were significantly associated with PIHCT acceptability after adjusted for other variables. A similar result was also reported from a population based study on routine testing in Botswana, where $81 \%$ of the study TB patients were extremely or very much in favor of routine HIV test. These results, seen with the high acceptability of PIHCT among TB patients (89.9\%) and $82.6 \%$ among non TB patients in this study, suggest that PIHCT is beneficial in improving access to testing and thereby increasing life-saving treatment users. HIV testing in combination with appropriate counseling is an important tool in the public health response to AIDS through providing Counseling and testing programs designed to promote knowledge of sero-status which can facilitate behavioral change, assist partners to negotiate safer sexual practice and allow early access to care, treatment and support for the HIV infected (Kasster William, 1997; Weiser et.al, 2006).

\section{CONCLUSIONS}

Despite high knowledge on HIV/TB, still there is misconception about disease transmission which requires provision of health education and expanding IEC to raise awareness on the disease transmission in the study participants. Most of the study subjects have heard about PIHCT and are in favor of the service provided in the TB clinic and OPD for TB and non TB patients respectively. There was relatively high acceptability of PIHCT in this study, with TB patients more accepted than non TB patients, which can be taken as constructive in the HIV/AIDS prevention and control program. The key perceived facilitator and barrier for PIHCT willingness were health care provider initiation and thinking self as not being at risk respectively, which was in line with other studies report. 


\section{Emiru Adeba and Wakgari Deressa}

\section{ACKNOWLEDGEMENTS}

My heartfelt thanks go to the Ethiopian Public Health Association (EPHA) for funding this research without which it would not have materialized.

\section{REFERENCES}

ACORD. Shashemene urban development program (ETH/07) proposal 1999-2003, Dean Bridle House, 52 Horse ferry Road, London SWIP 2AF, Pp. 2-8.

Coetzee, D., Hilderbrand, K. and Goemacre, E. (2004). Integrating tuberculosis and HIV care in the primary care setting in South Africa. Tropical Medicine and International Health 9(6): A11-A15 supplement.

Demssie, M., Lindtjørn, B., Tegbaru, B. (2000). Human immunodeficiency virus infection in tuberculosis patients in Addis Ababa. Ethiopian Journal of Health Development 4(3): 277-282.

Fylkesnes, K., Siziya, S. (2004). A randomized trial on acceptability of voluntary HIV testing. Tropical Medicine and International Health 2(5): 566-572.

Girma S. (2008). Assessment of the uptake of provider initiated HIV testing and counseling among OPD clients with possible clinical sign of HIV infection in Addis Ababa, Ethiopia MPH thesis, School of Public Health Addis Ababa University.

Jerene, D., Endale, A., Lindtjorn, B. (2007). Acceptability of HIV counseling and testing among tuberculosis patients in Arba Minch,south Ethiopia. BMC International Health and Human Right 7(4):1-9.

Kasster William, J., Dillon Beth, A., and Haley, C. (1997). OnSite, rapid HIV testing with same say results and counseling. AIDS 11:1045-1053.

Knut, F., Alan, H., Catharina, R., Pascal, M.K. (1999). HIV counseling and testing overemphasized high acceptance rates a threat to confidentiality and the right not to know. AIDS 13: 2469-2474.

MOH. (2006). $6^{\text {th }}$ AIDS report in Ethiopia. Addis Ababa, September 2006.

$\mathrm{MOH}$. (2003). Health and health related indicators. Addis Ababa; $\mathrm{MOH}, 2003 / 04$

$\mathrm{MOH}$. (2007). Health and health related indicators. Addis Ababa; $\mathrm{MOH}, 2006 / 07$.
Sci. Technol. Arts Res. J., Jan-March 2014, 3(1): 116-121

$\mathrm{MOH}$. (2005). Manual of tuberculosis, leprosy and TB/HIV prevention and control programs. $\mathrm{MOH}$. 2005; $3^{\text {rd }}$ Edition.

$\mathrm{MOH}$. (2004). Technical document for $5^{\text {th }}$ report on AIDS in Ethiopia. Addis Ababa; MOH, June 2004

$\mathrm{MOH}$. (2002). Tuberculosis and leprosy prevention and Control national manual. Addis Ababa.

$\mathrm{MOH}$. (2007). Guideline for management of opportunistic infections and antiretroviral treatment in adolescents and adults in Ethiopia. Federal HIV/AIDS prevention and control office, MOH July 2007.

Seid, F. (2008). Assessment of the acceptance of provider initiated HIV counseling and testing among TB patients in Adama, MPH thesis, School of Public Health, Addis Ababa University.

UNAIDS (2004). Global reference group on HIVAIDS and human rights. UNAIDS MHO policy statement on HIV testing.

Weiser, S.D., Heisler, M., Leiter, K., Percy-de Korte, F., Tlou, S. (2006). Routine HIV testing in Botswana: A populationbased study on attitudes, practices, and human rights concerns. PLoS Medicine 3(7): e261.

WHO. (2003). Guidelines for implementing collaborative TB and HIV programme activity. Geneva.

WHO. (2004). HIV /TB clinical manual, Geneva.

WHO. (2005). Interim policy on collaborative TB/HIV activities (WHO/HTM/TB/2004.330). Geneva.

WHO. (2004). Pushing to rapidly scale up measures to fight TB and HIV (Press Release WHO/5). Geneva; WHO, January 2004.

WHO. (2000). Tuberculosis and sustainable development, the Stop TB Initiative 2000 Report (WHO/CDS/STB/ 2000.4).

WHO. (2005). Annual report on surveillance, planning and financing for tuberculosis control. Geneva.

Ya Duil, M., Dermot, M., Anthony, H. (2001). Tuberculosis case fatality rates in high HIV prevalence populations in Sub-Saharan Africa. AIDS 15: 145-153.

Zachariah, R., Spielmann, M.L., and Chinji, C. (2003). Voluntary counseling, HIV testing and adjunctive cotrimazole reduces mortality in tuberculosis patients in Thyolo, Malawi. AIDS 17: 1053-1061. 